\title{
BOUNDARY REGULARITY OF WEAK SOLUTIONS TO NONLINEAR ELLIPTIC OBSTACLE PROBLEMS
}

\author{
MENG JUNXIA AND CHU YUMING
}

Received 25 April 2005; Revised 10 September 2005; Accepted 14 September 2005

We study the boundary regularity of weak solutions to nonlinear obstacle problem with $C^{1, \beta}$-obstacle function, and obtain the $C_{\mathrm{loc}}^{1, \alpha}$ boundary regularity.

Copyright (c) 2006 M. Junxia and C. Yuming. This is an open access article distributed under the Creative Commons Attribution License, which permits unrestricted use, distribution, and reproduction in any medium, provided the original work is properly cited.

\section{Introduction}

We consider the following variational inequality:

$$
\begin{aligned}
& u \in \mathfrak{R}: \int_{\Omega} A(x, \nabla u) \cdot(\nabla v-\nabla u) d x \\
& \quad \geq \int_{\Omega} H(x, u, \nabla u)(v-u) d x+\int_{\Omega} F(x, u) \cdot(\nabla v-\nabla u) d x
\end{aligned}
$$

for all $v \in \mathfrak{R}=\left\{v \in W_{0}^{1, p}(\Omega), v \geq \psi\right.$ a.e. in $\left.\Omega\right\}$. Here $\Omega$ is a bounded domain in $R^{N}(N \geq 2)$ with Lipschitz boundary, $2 \leq p \leq N$.

$A(x, \xi): \Omega \times R^{N} \rightarrow R^{N}$ satisfies the following conditions:

(i) $A$ is a vector valued function, the mapping $x \mapsto A(x, \xi)$ is measurable for all $\xi \in$ $R^{N}, \xi \mapsto A(x, \xi)$ is continuous for a.e. $x \in \Omega$;

(ii) the homogeneity condition: $A(x, t \xi)=t|t|^{p-2} A(x, \xi), t \in R, t \neq 0$;

(iii) the monotone inequality: $(A(x, \xi)-A(x, \zeta))(\xi-\zeta) \geq a|\xi-\zeta|^{p}$;

(iv) $|h|\left|a^{i j}\right|+\left|\partial A^{i}(x, h) / \partial x_{j}\right| \leq \tau_{1}|h|^{p-1}$;

(v) $\sum_{i, j=1}^{N} a^{i j} \xi_{i} \xi_{j} \geq \tau_{2}|h|^{p-2}|\xi|^{2}$;

(vi) $|A(x, \xi)-A(y, \xi)| \leq b_{1}\left(1+|\xi|^{p-1}\right)|x-y|^{\alpha_{0}}$;

(vii) $|A(x, \xi)-A(x, \eta)| \leq\left. b_{2}|| \xi\right|^{p-2} \xi-|\eta|^{p-2} \eta \mid$;

where $a^{i j}=\partial A^{i} / \partial h_{j}, a, b_{1}, b_{2}, \tau_{1}, \tau_{2}$ are positive constants. 
We assume that $H(x, u, \lambda), F(x, u)=\left\{F_{i}(x, u)\right\}_{1 \leq i \leq N}$ in (1.1) are of the form:

$$
\begin{gathered}
|H(x, u, \nabla u)| \leq c\left(|\nabla u|^{p / r^{\prime}}+|u|^{r-1}+g(x)\right), \\
|F(x, u)| \leq c\left(|u|^{q / p^{\prime}}+h(x)\right),
\end{gathered}
$$

where $p<q<r, r^{\prime}=r /(r-1), p^{\prime}=p /(p-1)$, and if $2 \leq p<N, r=N p /(N-p)$, while if $p=N$, then $r$ can be some sufficiently large positive number.

Higher regularity of the weak solution to the $p$-Laplacian obstacle problem

$$
I(u)=\inf \left\{\int_{\Omega}|\nabla u|^{p} d x: u \in K(\psi)\right\}
$$

where

$$
K(\psi)=\left\{v \in W^{1, p}(\Omega): v \geq \psi \text { a.e. }\right\}
$$

has been studied by various authors. In the case when $\psi$ is assumed to have only minimal regularity properties, it was shown by $[8,11]$ that the solution of $(1.1)$ is continuous. In particular, if $\psi \in C^{0, \alpha}(\Omega)$, then the solution $u$ is also an element of $C^{0, \alpha^{\prime}}(\Omega)$. In the case when $\psi \in C^{2}(\Omega)$, papers $[4,6,10,12]$ employed different techniques to prove interior $C^{1, \alpha}(\Omega)$ regularity for the solution $u$ to (1.4). Reference [1] gave an interesting result: the condition for $\mathfrak{R}$ to be nonempty is just that $\psi$ should have finite capacity. This implies, among other things, that $\psi^{+}=\max (\psi, 0)$ must vanish on $\partial \Omega, C$-almost everywhere. This condition is important for the existence of weak solutions to obstacle problem.

When $\psi$ is smooth (say $C^{1, \alpha}(\Omega)$ ), the interior regularity of weak solutions to problem (1.1) has been studied extensively by many authors $([3,13,14])$.

In view of De Giorgi class, paper [2] obtained $C^{0, \alpha}$ interior regularity for solutions of nonlinear elliptic obstacle problem with natural growth in the gradient by taking appropriate test function.

The main concern of these papers is the question of the regularity of the solution $u$ in terms of the given regularity properties of the obstacle $\psi$ and relevant data. This is especially interesting in view of the fact that there is a limit to the amount of regularity that $u$ can inherit from $\psi$ : it is possible for $\psi$ to be real analytic, but $u$ will be at best $C^{1,1}$, that is, have bounded second derivatives.

This paper obtains $C_{\text {loc }}^{1, \alpha}$ boundary regularity of weak solutions to the obstacle problem with $C^{1, \beta}$-obstacle function under controllable growth condition (1.2). We present a new proof to a useful comparison principle.

\section{Notations and preliminaries}

$\Omega$ is an open bounded subset of $R^{N}, N \geq 2 ; \partial \Omega$ is the boundary of $\Omega$. If $z \in R^{N}$, we put

$$
\begin{array}{cc}
B_{R}(z)=\left\{x \in R^{N}:|x-z|<R\right\}, & \Gamma_{R}(z)=\left\{x \in B_{R}(z): x_{n}=0\right\}, \\
B_{R}^{+}(z)=\left\{x \in B_{R}(z): x_{n}>0\right\}, & B_{R}^{-}(z)=\left\{x \in B_{R}(z): x_{n}<0\right\} .
\end{array}
$$

We denote by $B, B^{+}, B^{-}, \Gamma$, respectively, $B_{1}(0), B_{1}^{+}(0), B_{1}^{-}(0), \Gamma_{1}(0)$. For every set $E$ we denote by $\bar{E}$ its closure, and by $|E|$ its Lebesgue measure. $(f)_{R}=\left(1 /\left|B_{R}\right|\right) \int_{B_{R}} f(x) d x$. The 
letter $\mathrm{c}$ is used throughout to denote a positive constant, not necessarily the same at each occurrence.

Since $\bar{\Omega}$ is compact, $\partial \Omega$ can be covered by a finite number of neighbourhoods $V$ of its points. It is enough to prove the better regularity of $u$ holds true in $V \cap \Omega$. Since $\partial \Omega$ is a Lipschitz boundary, one can find $T$ which is an invertible Lipschitz mapping such that

$$
T(V)=B, \quad T(V \cap \Omega)=B^{+}, \quad T(V \backslash \bar{\Omega})=B^{-}, \quad T(V \cap \partial \Omega)=\Gamma .
$$

Under the mapping $T$ the variational inequality in $\Omega$ is transformed to a variational inequality of the same form in $B^{+}$, for $\bar{u}=u \circ T^{-1}$ which satisfies

$$
\begin{aligned}
& \int_{B^{+}} \bar{A}(x, \nabla \bar{u}) \cdot(\nabla v-\nabla \bar{u}) d x \\
& \quad \geq \int_{B^{+}} \bar{H}(x, \bar{u}, \nabla \bar{u})(v-\bar{u}) d x+\int_{B^{+}} \bar{F}(x, \bar{u}) \cdot(\nabla v-\nabla \bar{u}) d x, \quad \forall v \in \overline{\mathfrak{R}},
\end{aligned}
$$

where $\overline{\mathcal{R}}=\left\{v \in W_{0}^{1, p}\left(B^{+}\right), v \geq \psi\right.$, a.e. in $\left.B^{+}\right\}, \bar{A}, \bar{H}, \bar{F}$ satisfy assumptions of type (i)(vii), (1.2), (1.3) with different constants.

In order to simplify the notations, we still denote $\bar{u}, \overline{\mathfrak{R}} \bar{A}, \bar{H}, \bar{F}$ by $\mathcal{u}, \mathfrak{R}, A, H, F$, respectively.

Since the original $u \in W_{0}^{1, p}(\Omega)$, we define then

$$
u(x):= \begin{cases}u(x), & \text { if } x \in B^{+} \\ -3 u\left(x_{1}, \ldots, x_{n-1},-x_{n}\right)+4 u\left(x_{1}, \ldots, x_{n-1},-\frac{x_{n}}{2}\right), & \text { if } x \in B^{-}\end{cases}
$$

In light of Extension theorem [5, page 254], we only need to prove a better regularity of $u$ in $B^{+}$.

Definition 2.1. The function $u \in \mathfrak{R}$ that satisfies (2.3) for all $v \in \mathfrak{R}$ is called a weak solution to the obstacle problem with obstacle $\psi$.

Definition 2.2. Call $f \in C^{0, \alpha}(\Gamma)$, if for all $x \in \Gamma$, there exists $B_{r}(x)$ (a ball centered at $x$ of radius $r), r>0$, such that $f \in C^{0, \alpha}\left(B_{r}(x)\right)$.

In the sequel, we will abbreviate $B^{+} \cap B_{R}\left(y_{0}\right)=B_{R}^{+}, B^{+} \cap B_{\rho}\left(y_{0}\right)=B_{\rho}^{+}$, for $0<\rho<R \leq$ 1 , the point $y_{0} \in \Gamma$ to be understood.

In the following, we will use some lemmas which we state below.

Lemma 2.3. Let $w \in W^{1, p}\left(B_{R}^{+}\right)$be a solution of the Dirichlet problem

$$
\begin{gathered}
\int_{B_{R}^{+}} A(x, \nabla w) \nabla \phi d x=0 \quad \forall \phi \in W_{0}^{1, p}\left(B_{R}^{+}\right), \\
w-u \in W_{0}^{1, p}\left(B_{R}^{+}\right) .
\end{gathered}
$$


4 Boundary regularity

For $0<\rho<R / 2, \sigma \in(0,1)$,

$$
\begin{gathered}
\int_{B_{R}^{+}}|\nabla w|^{p} d x \leq c \int_{B_{R}^{+}}|\nabla u|^{p} d x \\
\int_{B_{\rho}^{+}}|\nabla w|^{p} d x \leq c\left(\frac{\rho}{R}\right)^{N} \int_{B_{R}^{+}}|\nabla w|^{p} d x \\
\int_{B_{\rho}^{+}}\left|\nabla w-(\nabla w)_{\rho}\right|^{p} d x \leq c\left(\frac{\rho}{R}\right)^{N+\sigma} \int_{B_{R}^{+}}\left|\nabla w-(\nabla w)_{R}\right|^{p} d x .
\end{gathered}
$$

Proof. We can easily get (2.6) by inserting $\phi=w-u$ in (2.5).

An argument similar to the one in [15, Lemma 2.2] shows that (2.7) hold.

The proof of (2.8) is similar to that of [9, Theorem 1.7].

Lemma 2.4. Let $v \in W^{1, p}\left(B_{R}^{+}\right)$be a solution of the Dirichlet problem

$$
\begin{gathered}
\int_{B_{R}^{+}} A(x, \nabla v) \nabla \phi d x=\int_{B_{R}^{+}} A(x, \nabla \psi) \nabla \phi d x, \\
w-v \in W_{0}^{1, p}\left(B_{R}^{+}\right), \quad \forall \phi \in W_{0}^{1, p}\left(B_{R}^{+}\right),
\end{gathered}
$$

then

$$
\begin{gathered}
\int_{B_{R}^{+}}|\nabla w|^{p} d x \leq c \int_{B_{R}^{+}}|\nabla v|^{p} d x, \\
\int_{B_{R}^{+}}|\nabla v-\nabla w|^{p} d x \leq c \int_{B_{R}^{+}}|\nabla \psi|^{p} d x .
\end{gathered}
$$

Proof. Formula (2.10) follows immediately from taking $\phi=w-v$ in (2.5).

Inserting $\phi=v-w$ in (2.5) and (2.9), by monotone inequality (iii) and Hölder's inequality, we have

$$
\begin{aligned}
\int_{B_{R}^{+}}|\nabla v-\nabla w|^{p} d x & \leq c \int_{B_{R}^{+}}(A(x, \nabla v)-A(x, \nabla w)) \cdot(\nabla v-\nabla w) d x \\
& =c \int_{B_{R}^{+}} A(x, \nabla \psi) \cdot(\nabla v-\nabla w) d x \\
& \leq c \int_{B_{R}^{+}}|\nabla \psi|^{p-1}|\nabla v-\nabla w| d x \\
& \leq c\left(\int_{B_{R}^{+}}|\nabla \psi|^{p} d x\right)^{(p-1) / p}\left(\int_{B_{R}^{+}}|\nabla v-\nabla w|^{p} d x\right)^{1 / p}
\end{aligned}
$$

from which we get (2.11).

Lemma 2.5. If $v \in W^{1, p}\left(B_{R}^{+}\right)$is a solution of the Dirichlet problem (2.9), then $v \geq \psi$ in $B_{R}^{+}$.

Proof. It follows from $v=u$ on $\partial B_{R}^{+}, u \in \mathfrak{R}$, that $v \geq \psi$ on $\partial B_{R}^{+}$. Let $\xi=\min (v, \psi), \xi=\psi$ on $\partial B_{R}^{+}, \xi-\psi \in W_{0}^{1, p}\left(B_{R}^{+}\right)$. As test functions in (2.9) we take $\phi=\xi-\psi$, from (2.9) and 
monotony inequality (iii), we have

$$
\begin{aligned}
0 & =\int_{B_{R}^{+}} A(x, \nabla v)-A(x, \nabla \psi) \cdot \nabla(\xi-\psi) d x \\
& =\int_{B_{R}^{+} \bigcap\{x, v(x) \leq \psi(x)\}} A(x, \nabla v)-A(x, \nabla \psi) \cdot \nabla(v-\psi) d x \\
& \geq a \int_{B_{R}^{+} \cap\{x, v(x) \leq \psi(x)\}}|\nabla v-\nabla \psi|^{p} d x \\
& =a \int_{B_{R}^{+}}|\nabla \xi-\nabla \psi|^{p} d x
\end{aligned}
$$

therefore $\xi=\psi$ a.e. in $B_{R}^{+}$, that is, $v \geq \psi$ a.e. in $B_{R}^{+}$.

This lemma is a useful comparison principle, it can be used to obtain the existence or regularity of solutions to elliptic equation or variational inequality.

We extend $v$ to $B^{+}$by setting $v=u$ on $B^{+} \backslash B_{R}^{+}$, and hence $v \in \mathfrak{R}$. We have the following corollary.

Corollary 2.6. Suppose $u$ is a weak solution to the obstacle problem (2.3), $v \in W^{1, p}\left(B_{R}^{+}\right)$ is a solution of the Dirichlet problem (2.9), then $v \in \mathfrak{R}$ satisfies the variational inequality

$$
\int_{B^{+}} A(x, \nabla u) \cdot(\nabla v-\nabla u) d x \geq \int_{B^{+}} H(x, u, \nabla u)(v-u) d x+\int_{B^{+}} F(x, u) \cdot(\nabla v-\nabla u) d x .
$$

Lemma 2.7. Assume $u$ is a weak solution to the obstacle problem (2.3), where $H$, F verify (1.2), (1.3), respectively, $g \in L^{t}\left(B^{+}\right)$with $t>N / p, h \in L^{s}\left(B^{+}\right)$with $s>p^{\prime}$, v satisfies (2.9), then

$$
\begin{aligned}
\int_{B_{R}^{+}}|\nabla v|^{p} d x \leq c[ & \left.\int_{B_{R}^{+}}|\nabla u|^{p} d x+\int_{B_{R}^{+}}|\nabla \psi|^{p} d x\right], \\
\int_{B_{R}^{+}}|\nabla u-\nabla v|^{p} d x \leq c\{ & {\left[\int_{B_{R}^{+}}\left(|\nabla u|^{p}+|u|^{r}\right) d x\right]^{1+\delta} } \\
+ & \left(\int_{B_{R}^{+}}|\nabla u|^{p} d x\right)^{q / p}+\left(\int_{B_{R}^{+}}|u|^{r} d x\right)^{q / p} \\
+ & \left.R^{N p(1-1 / r-1 / t) /(p-1)}+R^{N\left(1-p^{\prime} / s\right)}+R^{N(1-p / m)}\right\},
\end{aligned}
$$

where $\delta=(r-p) / r(p-1)>0$.

Proof. By inserting $\phi=v-u$ in (2.9), an application of Hölder's inequality and Young's inequality yields

$$
\begin{aligned}
\int_{B_{R}^{+}}|\nabla v|^{p} d x & \leq c \int_{B_{R}^{+}} A(x, \nabla v) \cdot \nabla v d x \\
& =c\left[\int_{B_{R}^{+}} A(x, \nabla v) \cdot \nabla u d x+\int_{B_{R}^{+}} A(x, \nabla \psi) \cdot \nabla(v-u) d x\right]
\end{aligned}
$$


Boundary regularity

$$
\begin{aligned}
\leq & c\left[\int_{B_{R}^{+}}|\nabla v|^{p-1}|\nabla u| d x+\int_{B_{R}^{+}}|\nabla \psi|^{p-1}|\nabla u-\nabla v| d x\right] \\
\leq & c\left\{\left(\int_{B_{R}^{+}}|\nabla v|^{p} d x\right)^{(p-1) / p}\left(\int_{B_{R}^{+}}|\nabla u|^{p} d x\right)^{1 / p}\right. \\
& \left.\quad+\left(\int_{B_{R}^{+}}|\nabla \psi|^{p} d x\right)^{(p-1) / p}\left(\int_{B_{R}^{+}}|\nabla u-\nabla v|^{p} d x\right)^{1 / p}\right\} \\
\leq & c \epsilon_{1} \int_{B_{R}^{+}}|\nabla v|^{p} d x+c\left(\epsilon_{1}, p\right) \int_{B_{R}^{+}}|\nabla u|^{p} d x+c \epsilon_{2} \int_{B_{R}^{+}}|\nabla u-\nabla v|^{p} d x \\
& +c\left(\epsilon_{2}, p\right) \int_{B_{R}^{+}}|\nabla \psi|^{p} d x \\
\leq & \left(c \epsilon_{1}+c \epsilon_{2}\right) \int_{B_{R}^{+}}|\nabla v|^{p} d x+\left(c\left(\epsilon_{1}, p\right)+c \epsilon_{2}\right) \int_{B_{R}^{+}}|\nabla u|^{p} d x \\
& +c\left(\epsilon_{2}, p\right) \int_{B_{R}^{+}}|\nabla \psi|^{p} d x
\end{aligned}
$$

for $\left(c \epsilon_{1}+c \epsilon_{2}\right)$ sufficiently small $\left(c \epsilon_{1}+c \epsilon_{2}<1\right)$, we can get $(2.15)$.

By $\psi \in W^{1, m}(\Omega), m>N$, we have

$$
\int_{B_{R}}|\nabla \psi|^{p} d x \leq c\|\nabla \psi\|_{m}^{p} R^{N(1-p / m)}
$$

Combining monotone inequality (iii), (2.9), (2.14), and (1.2) and using Poincare's inequality, Hölder's inequality, we have

$$
\begin{aligned}
\int_{B_{R}^{+}} \mid \nabla & u-\left.\nabla v\right|^{p} d x \\
\leq & c \int_{B_{R}^{+}}[A(x, \nabla u)-A(x, \nabla v)] \cdot(\nabla u-\nabla v) d x \\
\leq & c \int_{B_{R}^{+}}[H(x, u, \nabla u)(u-v)+(F(x, u)-A(x, \nabla \psi)) \cdot(\nabla u-\nabla v)] d x \\
\leq & c \int_{B_{R}^{+}}\left(|\nabla u|^{p(1-1 / r)}+|u|^{r-1}+|g|\right)|u-v| d x \\
& +c \int_{B_{R}^{+}}\left(|u|^{q / p^{\prime}}+h(x)\right)|\nabla u-\nabla v| d x+c \int_{B_{R}^{+}}|\nabla \psi|^{p-1} \cdot|\nabla u-\nabla v| d x \\
\leq & c\left[\int_{B_{R}^{+}}\left(|\nabla u|^{p}+|u|^{r}+|g|^{r /(r-1)}\right) d x\right]^{1-1 / r}\left(\int_{B_{R}^{+}}|u-v|^{r} d x\right)^{1 / r} \\
& +\left(\int_{B_{R}^{+}}\left(|u|^{q}+|h|^{p^{\prime}}\right) d x\right)^{1 / p^{\prime}}\left(\int_{B_{R}^{+}}|\nabla u-\nabla v|^{p} d x\right)^{1 / p} \\
& +\int_{B_{R}^{+}}|\nabla \psi|^{p-1} \cdot|\nabla u-\nabla v| d x
\end{aligned}
$$




$$
\begin{aligned}
& \leq c\left\{\left[\int_{B_{R}^{+}}\left(|\nabla u|^{p}+|u|^{r}\right) d x\right]^{1-1 / r}+\|g\|_{t} R^{N(1-1 / r-1 / t)}\right\} \\
& \quad \times R^{1-N(1 / p-1 / r)}\left(\int_{B_{R}^{+}}|\nabla u-\nabla v|^{p} d x\right)^{1 / p} \\
& \quad+c\left\{\left(\int_{B_{R}^{+}}\left(|u|^{p}+|\nabla u|^{p}\right) d x\right)^{q / p p^{\prime}}+\|h\|_{s} R^{N\left(1 / p^{\prime}-1 / s\right)}+\left\||\nabla \psi|^{p-1}\right\|_{p /(p-1)}\right\} \\
& \quad \times\left(\int_{B_{R}^{+}}|\nabla u-\nabla v|^{p} d x\right)^{1 / p}
\end{aligned}
$$

since $0<R \leq 1$, by (2.18), Hölder inequality, Young inequality, we have

$$
\begin{aligned}
& \int_{B_{R}^{+}}|\nabla u-\nabla v|^{p} d x \\
& \leq c\left\{\left[\int_{B_{R}^{+}}\left(|\nabla u|^{p}+|u|^{r}\right) d x\right]^{1+\delta}+\left(\int_{B_{R}^{+}}|\nabla u|^{p} d x\right)^{q / p}\right. \\
& +\left(\int_{B_{R}^{+}}|u|^{p} d x\right)^{q / p}+\|g\|_{t}^{p /(p-1)} R^{N p(1-1 / r-1 / t) /(p-1)} \\
& \left.+\|h\|_{s}^{p /(p-1)} R^{N\left(1-p^{\prime} / s\right)}+\int_{B_{R}^{+}}|\nabla \psi|^{p} d x\right\} \\
& \leq c\left\{\left[\int_{B_{R}^{+}}\left(|\nabla u|^{p}+|u|^{r}\right) d x\right]^{1+\delta}+\left(\int_{B_{R}^{+}}|\nabla u|^{p} d x\right)^{q / p}\right. \\
& \left.+\left(\int_{B_{R}^{+}}|u|^{p} d x\right)^{q / p}+R^{N p(1-1 / r-1 / t) /(p-1)}+R^{N\left(1-p^{\prime} / s\right)}+R^{N(1-p / m)}\right\} \\
& \leq c\left\{\left[\int_{B_{R}^{+}}\left(|\nabla u|^{p}+|u|^{r}\right) d x\right]^{1+\delta}+\left(\int_{B_{R}^{+}}|\nabla u|^{p} d x\right)^{q / p}\right. \\
& \left.+\left(\int_{B_{R}^{+}}|u|^{r} d x\right)^{q / r}\left|B_{R}\right|^{(q / p)(1-p / r)}+R^{N p(1-1 / r-1 / t) /(p-1)}+R^{N\left(1-p^{\prime} / s\right)}+R^{N(1-p / m)}\right\} \\
& \leq c\left\{\left[\int_{B_{R}^{+}}\left(|\nabla u|^{p}+|u|^{r}\right) d x\right]^{1+\delta}+\left(\int_{B_{R}^{+}}|\nabla u|^{p} d x\right)^{q / p}\right. \\
& \left.+\left(\int_{B_{R}^{+}}|u|^{r} d x\right)^{q / p}+R^{N(q / p)}+R^{N p(1-1 / r-1 / t) /(p-1)}+R^{N\left(1-p^{\prime} / s\right)}+R^{N(1-p / m)}\right\} \\
& \leq c\left\{\left[\int_{B_{R}^{+}}\left(|\nabla u|^{p}+|u|^{r}\right) d x\right]^{1+\delta}+\left(\int_{B_{R}^{+}}|\nabla u|^{p} d x\right)^{q / p}\right. \\
& \left.+\left(\int_{B_{R}^{+}}|u|^{r} d x\right)^{q / p}+R^{N p(1-1 / r-1 / t) /(p-1)}+R^{N\left(1-p^{\prime} / s\right)}+R^{N(1-p / m)}\right\}
\end{aligned}
$$

which implies (2.16). 


\section{3. $C^{0, \lambda}$ regularity}

Theorem 3.1. Assume that $H(x, u, \nabla u)$ satisfies (1.2), $g \in L^{t}\left(B^{+}\right)$with $t>N / p, F(x, u)$ satisfies (1.3), $h \in L^{s}\left(B^{+}\right)$with $s>N /(p-1)$, and $\psi \in W^{1, m}\left(B^{+}\right)$with $m>N$. If $u \in \mathfrak{R}$ makes (2.3) hold, then $u \in C^{0, \lambda}(\Gamma)$ with $\lambda=\min \{1-N(1 / t+1 / r-1 / p) /(p-1), 1-N /$ $s(p-1), 1-N / m\}$.

Before proceeding with the formal proof, we make an important observation. It is a well-known result.

Proposition 3.2. If $f \in W^{1, p}(\Omega)$, then for all constants $k \in R^{N}$,

$$
\int_{B_{\rho}}\left|\nabla f-(\nabla f)_{\rho, x_{0}}\right|^{p} d x \leq C(p) \int_{B_{\rho}}|\nabla f-k|^{p} d x
$$

for every $\rho$ for which $B_{\rho}\left(x_{0}\right) \subset \Omega$.

Proof. By elementary inequality, we have

$$
\int_{B_{\rho}}\left|\nabla f-(\nabla f)_{\rho, x_{0}}\right|^{p} d x \leq C(p)\left[\int_{B_{\rho}}|\nabla f-k|^{p} d x+\int_{B_{\rho}}\left|k-(\nabla f)_{\rho, x_{0}}\right|^{p} d x\right] .
$$

Moreover

$$
\begin{aligned}
\int_{B_{\rho}}\left|k-(\nabla f)_{\rho, x_{0}}\right|^{p} d x & =\left|B_{\rho}\right|\left|k-(\nabla f)_{\rho, x_{0}}\right|^{p}=\left|B_{\rho}\right|\left|k-\frac{1}{\left|B_{\rho}\right|} \int_{B_{\rho}} \nabla f d x\right|^{p} \\
& =\left|B_{\rho}\right|\left|\frac{1}{\left|B_{\rho}\right|} \int_{B_{\rho}}(k-\nabla f) d x\right|^{p}=\left|B_{\rho}\right|^{1-p}\left|\int_{B_{\rho}}(k-\nabla f) d x\right|^{p} \\
& \leq\left|B_{\rho}\right|^{1-p} \int_{B_{\rho}}|\nabla f-k|^{p} d x\left|B_{\rho}\right|^{p(1-1 / p)}=\int_{B_{\rho}}|\nabla f-k|^{p} d x .
\end{aligned}
$$

Therefore (3.1) holds for any $k \in R^{N}$.

Proof of Theorem 3.1. To get the regularity, we need to prove the following inequality:

$$
\int_{B_{\rho}^{+}}|\nabla u|^{p} d x \leq c \rho^{N-p+p \lambda}
$$

Let us consider three different situations.

(1) If $B_{2 R}\left(y_{0}\right) \subset B^{+}$, inequality (3.4)-(4.1) has been proved in [13], since it is related to interior regularity.

(2) If $B_{R}\left(y_{0}\right) \subset B^{-}$, by Extension theorem [5, page 254], if we can get $C^{1, \alpha}$ regularity of $u$ in $B^{+}$, we can deduce the same result for $u$ in $B^{-}$, so we need not care about this situation. 
(3) If $B_{R}\left(y_{0}\right) \cap B^{+} \neq \varnothing$, we also give three different situations as follows:
(a) $y_{0} \in \Gamma$,
(b) $y_{0} \in B^{-}$,
(c) $y_{0} \in B^{+}$.

We only prove the situation (a), since the others can be transformed into the situation (a) or the interior regularity situation by applying the finitely covered theorem, see [13]. Assume $h \in L^{s}\left(B^{+}, R^{N}\right)$ with $s>N /(p-1), \psi \in W^{1, m}\left(B^{+}\right)$with $m>N$, we see that

$$
\begin{aligned}
& \int_{B_{R}^{+}}|h|^{p /(p-1)} \leq\|h\|_{s}^{p /(p-1)} R^{N[1-p / s(p-1)]}, \\
& \left.\int_{B_{R}^{+}}|| \nabla \psi\right|^{p-2} \nabla \psi-\left.\left(|\nabla \psi|^{p-2} \nabla \psi\right)_{R}\right|^{p /(p-1)} d x \leq c \int_{B_{R}^{+}}|\nabla \psi|^{p} d x \leq c\|\nabla \psi\|_{m}^{p} R^{N(1-p / m)} .
\end{aligned}
$$

Combining (2.7), (2.10), (2.11), (2.16), and (3.5), we have

$$
\begin{aligned}
& \int_{B_{\rho}^{+}}|\nabla u|^{p} d x \\
& \leq c \int_{B_{\rho}^{+}}|\nabla w|^{p} d x+c \int_{B_{\rho}^{+}}|\nabla u-\nabla w|^{p} d x \\
& \leq c\left(\frac{\rho}{R}\right)^{N} \int_{B_{R}^{+}}|\nabla u|^{p} d x+c \int_{B_{R}^{+}}|\nabla u-\nabla v|^{p} d x+c \int_{B_{R}^{+}}|\nabla v-\nabla w|^{p} d x \\
& \leq c\left(\frac{\rho}{R}\right)^{N} \int_{B_{R}^{+}}|\nabla u|^{p} d x \\
& +c\left\{\left[\int_{B_{R}^{+}}\left(|\nabla u|^{p}+|u|^{r}\right) d x\right]^{1+\delta}+\left(\int_{B_{R}^{+}}|\nabla u|^{p} d x\right)^{q / p}+\left(\int_{B_{R}^{+}}|u|^{r} d x\right)^{q / p}\right. \\
& \left.+R^{N p(1-1 / r-1 / t) /(p-1)}+R^{N[1-p / s(p-1)]}+R^{N(1-p / m)}\right\} \\
& \leq c\left(\frac{\rho}{R}\right)^{N} \int_{B_{R}^{+}}|\nabla u|^{p} d x+c\left[\int_{B_{R}^{+}}\left(|\nabla u|^{p}+|u|^{r}\right) d x\right]^{1+\delta} \\
& +c\left(\int_{B_{R}^{+}}|\nabla u|^{p} d x\right)^{q / p}+c\left(\int_{B_{R}^{+}}|u|^{r} d x\right)^{q / p}+c R^{N-p+p \lambda},
\end{aligned}
$$

where $\lambda=\min \{1-N(1 / t+1 / r-1 / p) /(p-1), 1-N / s(p-1), 1-N / m\}$.

By $t>N / p$, we have the following.

(i) If $2 \leq p<N$, then $1 / t<p / N, 1 / t+1 / r-1 / p<p / N+(N-p) / N p-1 / p=(p-1) / N$, $N(1 / t+1 / r-1 / p) /(p-1)<1$.

(ii) If $p=N$, by $t>1$, we can assume that $r$ is a positive number sufficiently large, such that: $1 / t+1 / r<1$, so $N(1 / t+1 / r-1 / N) /(N-1)<(N /(N-1))(1-1 / N)=1$.

Hence, if $2 \leq p \leq N$, we always have $N(1 / t+1 / r-1 / p) /(p-1)<1$.

Using $s>N /(p-1), m>N$, by the definition of $\lambda$, we see that: $0<\lambda<1$. 
In the meantime, by Poincare's inequality and Hölder's inequality, we also have

$$
\begin{aligned}
\int_{B_{\rho}^{+}}|u|^{r} d x & \leq c \int_{B_{\rho}^{+}}\left|u_{R}\right|^{r} d x+c \int_{B_{R}^{+}}\left|u-u_{R}\right|^{r} d x \\
& \leq c\left(\frac{\rho}{R}\right)^{N} \int_{B_{R}^{+}}|u|^{r} d x+c R^{r[1-N(1 / p-1 / r)]}\left(\int_{B_{R}^{+}}|\nabla u|^{p} d x\right)^{r / p},
\end{aligned}
$$

where

$$
r\left[1-N\left(\frac{1}{p}-\frac{1}{r}\right)\right]= \begin{cases}\frac{N p}{N-p}\left[1-N\left(\frac{1}{p}-\frac{N-p}{N p}\right)\right]=0, & \text { if } 2 \leq p<N \\ r\left[1-N\left(\frac{1}{N}-\frac{1}{r}\right)\right]=N, & \text { if } p=N .\end{cases}
$$

Adding (3.7) to (3.6) and setting

$$
\phi(R)=\int_{B_{R}^{+}}\left(|\nabla u|^{p}+|u|^{r}\right) d x
$$

we obtain

$$
\phi(\rho) \leq c\left[\left(\frac{\rho}{R}\right)^{N}+\chi(R)\right] \phi(R)+c R^{N-p+p \lambda}
$$

where

$$
\begin{aligned}
\chi(R)= & {\left[\int_{B_{R}^{+}}\left(|\nabla u|^{p}+|u|^{r}\right) d x\right]^{\delta} } \\
& +\left(\int_{B_{R}^{+}}|\nabla u|^{p} d x\right)^{(q-p) / p}+\left(\int_{B_{R}^{+}}|u|^{r} d x\right)^{(q-p) / p} \\
& + \begin{cases}\left(\int_{B_{R}^{+}}|\nabla u|^{p} d x\right)^{(r-p) / p}, & \text { if } 2 \leq p<N \\
R^{N}\left(\int_{B_{R}^{+}}|\nabla u|^{p} d x\right)^{(r-p) / p}, & \text { if } p=N .\end{cases}
\end{aligned}
$$

We can always get $\chi(R) \rightarrow 0$ as $R \rightarrow 0^{+}$. Applying [7, page 86, Lemma 2.1], we deduce that for $\rho$ sufficiently small,

$$
\int_{B_{\rho}^{+}}|\nabla u|^{p} d x \leq \phi(\rho) \leq c \rho^{N-p+p \lambda}
$$

By Dirichlet growth theorem (see [7, page 64, Theorem 1.1]), $u \in C_{\text {loc }}^{0, \lambda}(\Gamma)$. 


\section{4. $C^{1, \alpha_{1}}$ regularity}

Theorem 4.1. Assume that $H(x, u, \nabla u)$ satisfies (1.2), $g \in L^{t}\left(B^{+}\right)$with $t>N, F$ $\in C^{0, \beta}\left(B^{+}, R\right)$ with $\beta>0, \psi \in C^{1, \gamma}\left(B^{+}\right)$for some $\gamma>0$. If $u \in \mathfrak{R}$ makes (2.3) hold, then $u \in C^{1, \alpha_{1}}(\Gamma)$ for some $0<\alpha_{1}<\sigma / p$.

To get our result, we need to prove the following inequality:

$$
\int_{B_{\rho}^{+}}\left|\nabla u-(\nabla u)_{\rho}\right|^{p} d x \leq c \rho^{N+p \alpha_{1}}
$$

It is easy to see that $|\nabla \psi|^{p-2} \nabla \psi \in C^{0, \gamma}\left(B^{+}\right)$if $\psi \in C^{1, \gamma}\left(B^{+}\right)$and $2 \leq p \leq N$.

Utilizing the conditions of Theorem 4.1, we see that:

$$
\begin{aligned}
& \int_{B_{R}^{+}}\left|F-F_{R}\right|^{p /(p-1)} d x \leq\|F\|_{C^{0, \beta}\left(B^{+}\right)}^{p /(p-1)} R^{N+\beta p /(p-1)}, \\
& |\nabla \psi(x)|^{p-2} \nabla \psi(x)-|\nabla \psi(y)|^{p-2}|\nabla \psi(y)| \\
& \quad \leq|||\nabla \psi|^{p-2} \nabla \psi \|_{C^{0, \gamma\left(B^{+}\right)}}|x-y|^{\gamma}, \quad \forall x, y \in B^{+} .
\end{aligned}
$$

By $\psi \in C^{1, \gamma}\left(B^{+}\right)$, we can get $|\nabla \psi|^{p-1} \leq c$, so combining condition (vi) we have

$$
|A(x, \nabla \psi)-A(y, \nabla \psi)| \leq c|x-y|^{\alpha_{0}} .
$$

By condition (vii), (4.3), and (4.4), we see that for all $\phi \in W_{0}^{1, p}\left(B_{R}^{+}\right)$, there holds

$$
\begin{aligned}
\int_{B_{R}^{+}} A(x, \nabla \psi) \cdot \nabla \phi d x \\
\quad=\int_{B_{R}^{+}}\left(A(x, \nabla \psi)-(A(x, \nabla \psi))_{R}\right) \cdot \nabla \phi d x \\
=\int_{B_{R}^{+}} f_{B_{R}}[A(x, \nabla \psi(x))-A(y, \nabla \psi(y))] \cdot \nabla \phi(x) d y d x \\
=\int_{B_{R}^{+}} f_{B_{R}}[A(x, \nabla \psi(x))-A(x, \nabla \psi(y)) \\
\quad \leq c \int_{B_{R}^{+}} f_{B_{R}}\left(|x-y|^{\alpha_{0}}+\left.|| \nabla \psi(x)\right|^{p-2} \nabla \psi(x)-|\nabla \psi(y)|^{p-2} \nabla \psi(y) \mid\right)|\nabla \phi(x)| d y d x \\
\leq c \int_{B_{R}^{+}}\left(R^{\alpha_{0}}+R^{\gamma}\right)|\nabla \phi(x)| d x .
\end{aligned}
$$

From last formula, we see that

$$
\int_{B_{R}^{+}} A(x, \nabla \psi) \cdot \nabla \phi d x \leq c \int_{B_{R}^{+}}\left(R^{\alpha_{0}}+R^{\gamma}\right)|\nabla \phi(x)| d x .
$$

In the following, we give two lemmas which will be used in the proof of Theorem 4.1. 
Lemma 4.2. Assume that $\psi \in C^{1, \gamma}\left(B^{+}\right), w, v \in W^{1, p}\left(B_{R}^{+}\right)$solve the Dirichlet problem (2.5), (2.9), respectively, then there holds

$$
\int_{B_{R}^{+}}|\nabla v-\nabla w|^{p} d x \leq c\left(R^{N+\gamma p /(p-1)}+R^{N+\alpha_{0} p /(p-1)}\right)
$$

Proof. Inserting $\phi=v-w$ in (2.5) and (2.9), by (4.6), monotone inequality (iii), and Hölder inequality, we have

$$
\begin{aligned}
\int_{B_{R}^{+}}|\nabla v-\nabla w|^{p} d x & \leq c \int_{B_{R}^{+}}(A(x, \nabla v)-A(x, \nabla w)) \cdot(\nabla v-\nabla w) d x \\
& =c \int_{B_{R}^{+}} A(x, \nabla \psi) \cdot(\nabla v-\nabla w) d x \\
& \leq c \int_{B_{R}^{+}}\left(R^{\alpha_{0}}+R^{\gamma}\right)|\nabla v-\nabla w| d x \\
& \leq c\left(R^{\gamma+N(p-1) / p}+R^{\alpha_{0}+N(p-1) / p}\right)\left(\int_{B_{R}^{+}}|\nabla v-\nabla w|^{p} d x\right)^{1 / p} .
\end{aligned}
$$

From last formula, we get (4.7).

Lemma 4.3. Assume that $A(x, \xi)$ satisfies condition ( $i)$-(vii), $u$ is a weak solution to obstacle problem (1.1), where $H$ verifies (1.2), $g \in L^{t}\left(B^{+}\right), t>N ; F \in C^{0, \beta}\left(B^{+}\right), \beta>0 ; \psi \in C^{1, \gamma}\left(B^{+}\right)$, $\gamma>0 . v \in W^{1, p}\left(B_{R}^{+}\right)$solves the Dirichlet problem (2.9), then there holds

$$
\begin{aligned}
\int_{B_{R}^{+}}|\nabla u-\nabla v|^{p} d x \leq c\{ & {\left[\int_{B_{R}^{+}}\left(|\nabla u|^{p}+|u|^{r}\right) d x\right]^{1+\delta}+R^{N p(1-1 / r-1 / t) /(p-1)} } \\
& \left.+R^{N+\beta p /(p-1)}+R^{N+\gamma p /(p-1)}+R^{N+\alpha_{0} p /(p-1)}\right\},
\end{aligned}
$$

where $\delta=(r-p) / r(p-1)>0$.

Proof. Combining monotone inequality (iii), and (1.2), (2.14), (2.18), (4.6), Hölder inequality, we have

$$
\begin{aligned}
\int_{B_{R}^{+}}|\nabla u-\nabla v|^{p} d x \leq & c \int_{B_{R}^{+}}[A(x, \nabla u)-A(x, \nabla v)] \cdot(\nabla u-\nabla v) d x \\
& \leq c \int_{B_{R}^{+}}[H(x, u, \nabla u)(u-v)+(F(x, u)-A(x, \nabla \psi)) \cdot(\nabla u-\nabla v)] d x \\
& \leq c \int_{B_{R}^{+}}\left(|\nabla u|^{p(1-1 / r)}+|u|^{r-1}+|g|\right)|u-v| d x \\
& +c \int_{B_{R}^{+}}\left|F-F_{R}\right||\nabla u-\nabla v| d x+c \int_{B_{R}^{+}}\left(R^{\alpha_{0}}+R^{\gamma}\right)|\nabla u-\nabla v| d x
\end{aligned}
$$




$$
\begin{aligned}
& \leq c\left\{\left(\int_{B_{R}^{+}}\left(|\nabla u|^{p}+|u|^{r}+|g|^{r /(r-1)}\right) d x\right)^{1-1 / r}\left(\int_{B_{R}^{+}}|u-v|^{r} d x\right)^{1 / r}\right. \\
& \quad+\left(\int_{B_{R}^{+}}\left|F-F_{R}\right|^{p^{\prime}} d x\right)^{1 / p^{\prime}}\left(\int_{B_{R}^{+}}|\nabla u-\nabla v|^{p} d x\right)^{1 / p} \\
& \left.\quad+\int_{B_{R}^{+}}\left(R^{\alpha_{0}}+R^{\gamma}\right)|\nabla u-\nabla v| d x\right\} \\
& \leq c\left\{\left[\int_{B_{R}^{+}}\left(|\nabla u|^{p}+|u|^{r}\right) d x\right]^{1-1 / r}+\|g\|_{t} R^{N(1-1 / r-1 / t)}\right\} \\
& \quad \times R^{1-N(1 / p-1 / r)}\left(\int_{B_{R}^{+}}|\nabla u-\nabla v|^{p} d x\right)^{1 / p} \\
& \quad+c\left\{\left(\int_{B_{R}^{+}}\left|F-F_{R}\right|^{p^{\prime}} d x\right)^{1 / p^{\prime}}+R^{\gamma+N(p-1) / p}+R^{\alpha_{0}+N(p-1) / p}\right\} \\
& \quad \times\left(\int_{B_{R}^{+}}|\nabla u-\nabla v|^{p} d x\right)^{1 / p} \cdot
\end{aligned}
$$

Since $0<R \leq 1$, by (4.2), and last formula, we have

$$
\begin{aligned}
\int_{B_{R}^{+}}|\nabla u-\nabla v|^{p} d x \leq c\{ & {\left[\int_{B_{R}^{+}}\left(|\nabla u|^{p}+|u|^{r}\right) d x\right]^{1+\delta}+R^{N p(1-1 / r-1 / t) /(p-1)} } \\
& \left.+R^{N+\beta p /(p-1)}+R^{N+\gamma p /(p-1)}+R^{N+\alpha_{0} \cdot p /(p-1)}\right\}
\end{aligned}
$$

Hence (4.9) holds.

Similar to the proof of (3.12), we get for any $\mu \in(0,1)$

$$
\int_{B_{\rho}^{+}}|\nabla u|^{p} d x, \quad \int_{B_{\rho}^{+}}|\nabla v|^{p} d x, \quad \int_{B_{\rho}^{+}}|u|^{r} d x \leq c \rho^{N-p+p \mu}
$$

In view of $t>N$, we have the following.

(i) If $2 \leq p<N, 1-1 / r-1 / t>1-1 / r-1 / N=(p-1) / p$, from which we get $N p(1-$ $1 / r-1 / t) /(p-1)>N$.

(ii) If $p=N$, we can assume that $r$ is a positive number large such that $1 / t+1 / r<$ $1 / N, N p(1-1 / r-1 / t) /(p-1)>N^{2}(1-1 / N) /(N-1)=N$.

Hence,we always have $N p(1-1 / r-1 / t) /(p-1)>N$ when $2 \leq p \leq N$.

In the following, we prove Theorem 4.1 . 
14 Boundary regularity

By (4.2), (4.3), (4.9), Lemmas 2.3, 4.2, and 4.3, we have

$$
\begin{aligned}
& \int_{B_{\rho}^{+}}\left|\nabla u-(\nabla u)_{\rho}\right|^{p} d x \\
& \leq c \int_{B_{\rho}^{+}}\left|\nabla u-(\nabla w)_{\rho}\right|^{p} d x \\
& \leq c \int_{B_{\rho}^{+}}\left|\nabla w-(\nabla w)_{\rho}\right|^{p} d x+c \int_{B_{\rho}^{+}}|\nabla u-\nabla v|^{p} d x+c \int_{B_{\rho}^{+}}|\nabla v-\nabla w|^{p} d x \\
& \leq c \int_{B_{\rho}^{+}}\left|\nabla w-(\nabla w)_{\rho}\right|^{p} d x \\
& +c\left\{\left[\int_{B_{R}^{+}}\left(|\nabla u|^{p}+|u|^{r}\right) d x\right]^{1+\delta}\right. \\
& \left.+R^{N p(1-1 / r-1 / t) /(p-1)}+R^{N+\beta p /(p-1)}+R^{N+\gamma p /(p-1)}+R^{N+\alpha_{0} p /(p-1)}\right\} \\
& +c\left(R^{N+\gamma p /(p-1)}+R^{N+\alpha_{0} p /(p-1)}\right) \\
& \leq c\left(\frac{\rho}{R}\right)^{N+\sigma} \int_{B_{R}^{+}}\left|\nabla u-(\nabla u)_{R}\right|^{p} d x \\
& +c\left\{\left[\int_{B_{R}^{+}}\left(|\nabla u|^{p}+|u|^{r}\right) d x\right]^{1+\delta}\right. \\
& \left.+R^{N p(1-1 / r-1 / t) /(p-1)}+R^{N+\beta p /(p-1)}+R^{N+\gamma p /(P-1)}+R^{N+\alpha_{0} p /(p-1)}\right\} \\
& \leq c\left(\frac{\rho}{R}\right)^{N+\sigma} \int_{B_{R}^{+}}\left|\nabla u-(\nabla u)_{R}\right|^{p} d x \\
& +c\left(R^{(1+\delta)(N-p+p \mu)}+R^{N p(1-1 / r-1 / t) /(p-1)}+R^{N+\beta p /(p-1)}\right. \\
& \left.+R^{N+\gamma p /(P-1)}+R^{N+\alpha_{0} p /(p-1)}\right) .
\end{aligned}
$$

We can select $\mu$ sufficiently close to 1 , such that $(1+\delta)(N-p+p \mu)>N$. Hence we get

$$
\int_{B_{\rho}^{+}}\left|\nabla u-(\nabla u)_{\rho}\right|^{p} d x \leq c\left(\frac{\rho}{R}\right)^{N+\sigma} \int_{B_{R}}\left|\nabla u-(\nabla u)_{R}\right|^{p} d x+c R^{N+p \alpha_{1}}
$$

for some $0<\alpha_{1}<\sigma / p$.

Applying [7, page 86, Lemma 2.1], we get

$$
\int_{B_{\rho}^{+}}\left|\nabla u-(\nabla u)_{\rho}\right|^{p} d x \leq c \rho^{N+p \alpha_{1}}
$$

for $\rho$ sufficiently small. By [7, page 72, Theorem 1.3], we obtain that $u \in C_{\text {loc }}^{1, \alpha_{1}}(\Gamma)$. 


\section{Acknowledgments}

We would like to thank professor Fang AiNong for many useful comments on the subject, the project supported by the Natural Science Foundation of Hebei University, the National Natural Science Foundation of China under Grant 10471039, and the Zhejiang Province Natural Science Foundation of China under Grant M103087.

\section{References}

[1] D. R. Adams, Capacity and the obstacle problem, Applied Mathematics and Optimization. An International Journal 8 (1982), no. 1, 39-57.

[2] F. Chiacchio, Regularity for solutions of nonlinear elliptic equations with natural growth in the gradient, Bulletin des Sciences Mathématiques 124 (2000), no. 1, 57-74.

[3] H. J. Choe, A regularity theory for a general class of quasilinear elliptic partial differential equations and obstacle problems, Archive for Rational Mechanics and Analysis 114 (1991), no. 4, 383-394.

[4] H. J. Choe and J. L. Lewis, On the obstacle problem for quasilinear elliptic equations of p Laplacian type, SIAM Journal on Mathematical Analysis 22 (1991), no. 3, 623-638.

[5] L. C. Evans, Partial Differential Equations, Graduate Studies in Mathematics, vol. 19, American Mathematical Society, Rhode Island, 1998.

[6] M. Fuchs, Hölder continuity of the gradient for degenerate variational inequalities, Nonlinear Analysis. Theory, Methods \& Applications. An International Multidisciplinary Journal. Series A: Theory and Methods 15 (1990), no. 1, 85-100.

[7] M. Giaquinta, Multiple Integrals in the Calculus of Variations and Nonlinear Elliptic Systems, Annals of Mathematics Studies, vol. 105, Princeton University Press, New Jersey, 1983.

[8] J. Heinonen and T. Kilpeläinen, On the Wiener criterion and quasilinear obstacle problems, Transactions of the American Mathematical Society 310 (1988), no. 1, 239-255.

[9] G. M. Lieberman, The natural generalization of the natural conditions of Ladyzhenskaya and Ural'tseva for elliptic equations, Communications in Partial Differential Equations 16 (1991), no. 2-3, 311-361.

[10] P. Lindqvist, Regularity for the gradient of the solution to a nonlinear obstacle problem with degenerate ellipticity, Nonlinear Analysis. Theory, Methods \& Applications. An International Multidisciplinary Journal. Series A: Theory and Methods 12 (1988), no. 11, 1245-1255.

[11] J. H. Michael and W. P. Ziemer, Interior regularity for solutions to obstacle problems, Nonlinear Analysis. Theory, Methods \& Applications. An International Multidisciplinary Journal. Series A: Theory and Methods 10 (1986), no. 12, 1427-1448.

[12] J. Mu, Higher regularity of the solution to the p-Laplacian obstacle problem, Journal of Differential Equations 95 (1992), no. 2, 370-384.

[13] Z. Tan and Z. Q. Yan, Regularity of weak solutions to some degenerate elliptic equations and obstacle problems, Northeastern Mathematical Journal. Dongbei Shuxue 9 (1993), no. 2, 143-156.

[14] J. Yang, Regularity of weak solutions to quasilinear elliptic obstacle problems, Acta Mathematica Scientia. Series B. English Edition 17 (1997), no. 2, 159-166.

[15] S. Z. Zheng, Partial regularity of A-harmonic systems of equations and quasiregular mappings, Chinese Annals of Mathematics. Series A. Shuxue Niankan. A Ji 19 (1998), no. 1, 63-72 (Chinese), translation in Chinese Journal of Contemporary Mathematics19 (1998), no. 1, 19-30.

Meng Junxia: College of Mathematics and Computer Science, Hebei University, Hebei 071002, China E-mail address: junxia@hbu.cn

Chu Yuming: College of Science, Huzhou University, Zhejiang 313000, China

E-mail address: chuyuming@hutc.zj.cn 